\title{
Urinary [TIMP-2] $\times$ [IGFBP7] and serum procalcitonin to predict and assess the risk for short-term outcomes in septic and non-septic critically ill patients
}

Ilaria Godi ${ }^{1} 2^{*}$, Silvia De Rosa ${ }^{1,3}$, Francesca Martino ${ }^{1,4}$, Simona Bazzano ${ }^{1,5}$, Marina Martin ${ }^{3}$, Elisa Boni ${ }^{3}$, Maria

Rosa Carta ${ }^{6}$, Claudia Tamayo Diaz ${ }^{1}$, Gaia Mari ${ }^{1}$, Anna Lorenzin ${ }^{1}$, Massimo de Cal ${ }^{1}$, Valentina Corradi ${ }^{1}$,

Carlotta Caprara ${ }^{1}$, Davide Giavarina ${ }^{6}$ and Claudio Ronco ${ }^{1,4}$

\begin{abstract}
Background: Biomarkers can play a critical role by facilitating diagnosis and stratification of disease, as well as assessment or prediction of disease severity. Urinary tissue inhibitor of metalloproteinase-2 and insulin-like growth factor binding protein 7 product ([TIMP-2] × [IGFBP7]) predict the development and progression of AKI and recently procalcitonin (PCT), a widely used biomarker for sepsis diagnosis and management, has been associated with AKI occurrence in ICU patients. To assess combinations of [TIMP-2] $\times$ [IGFBP7] and PCT results for prediction and risk stratification of short-term outcomes in septic and non-septic patients, a retrospective cohort analysis of critically ill patients was performed in a multidisciplinary ICU. ROC curve analysis was used in order to evaluate predictive performance of combined results of [TIMP-2] $\times$ [IGFBP7] and PCT at the time of admission for AKI development. To verify the utility of adding [TIMP-2] $\times$ [IGFBP7] and PCT results for risk assessment, we evaluated the predictive value of having a singlemarker positivity compared to a double-marker positivity using the widely used cut-off of $0.3(\mathrm{ng} / \mathrm{mL})^{2} / 1000$ for [TIMP-2] $\times$ [IGFBP7] and $0.5 \mu \mathrm{g} / \mathrm{L}$ for PCT. Risk assessment for AKI occurrence within $48 \mathrm{~h}$, acute kidney disease (AKD) and mortality at 7 days was performed by logistic/Cox regression analysis.
\end{abstract}

Results: 433 patients were analysed, of whom 168 had AKI within 48 h (93 septic and 65 non-septic patients). Combination of [TIMP-2] $\times$ [IGFBP7] and PCT showed a good predictive ability for AKI occurrence (AUC $0.81,95 \% \mathrm{Cl}$ $0.77-0.86, p<0.001$, Sens $78 \%$, Spec 73\%). Combinations of biomarkers increased the odd ratios (OR) considerably. A single-marker positivity showed a fourfold risk increase, while the double-marker positivity a 26-fold risk increase for AKI occurrence. Moreover, the double-marker positivity showed an elevated risk for AKD at 7 days in non-septic patients (OR 15.9, 95\% Cl 3,21-73,57, $p<0.001$ ) and for mortality within 7 days in septic patients (HR 4.1, 95\% Cl $1.4-11.8, p=0.001)$.

Conclusions: Although combining the results of [TIMP-2] $\times$ [IGFBP7] and PCT may be a useful tool to identify and stratify ICU patients at high risk for septic AKI and short-term adverse outcomes, data should be confirmed in a large prospective study.

Keywords: Acute kidney injury, Sepsis, Intensive care unit, [TIMP-2] × [IGFBP7], Procalcitonin

\footnotetext{
*Correspondence: ilaria.g88@libero.it

${ }^{2}$ Department of Medicine-DIMED, Section of Anesthesiology

and Intensive Care Medicine, University of Padova, Padua, Italy

Full list of author information is available at the end of the article
} adaptation, distribution and reproduction in any medium or format, as long as you give appropriate credit to the original author(s) and the source, provide a link to the Creative Commons licence, and indicate if changes were made. The images or other third party material in this article are included in the article's Creative Commons licence, unless indicated otherwise in a credit line to the material. If material is not included in the article's Creative Commons licence and your intended use is not permitted by statutory regulation or exceeds the permitted use, you will need to obtain permission directly from the copyright holder. To view a copy of this licence, visit http://creativeco mmons.org/licenses/by/4.0/. 


\section{Introduction}

Acute kidney Injury (AKI) occurs in 1 to $66 \%$ of hospitalized patients, depending on the economic status [1]. It is a frequent complication of serious acute illness and sepsis is one of the common causes [2]. AKI is associated with increased morbidity, mortality and high health costs $[3,4]$.

In this context, biomarkers may play a critical role by facilitating diagnosis and stratification of disease, as well as assessment or prediction of disease severity or response to therapy. Among the most recent ones, urinary tissue inhibitor of metalloproteinases-2 (TIMP-2) and insulin-like growth factor binding protein 7 (IGFBP7) product have been proven to be a good predictor of AKI [5] and received FDA approval for AKI risk assessment in critically ill patients. Recently, procalcitonin (PCT), a widely used biomarker for sepsis diagnosis and management, showed a significant association with AKI development [6]. Despite this association, the usefulness of adding PCT levels to existing models for predicting the occurrence of AKI in critically ill patients is still not clear [7]. Nevertheless, a combination of kidney-specific and non-kidney-specific biomarkers may contribute to early identification of AKI and stratification of critically ill patients. Here, we conducted this study to evaluate the relationship between combinations of [TIMP-2] $\times$ [IGFBP7] and PCT results and AKI in critically ill patients. Our hypothesis is that the admission [TIMP-2] $\times$ [IGFBP7]/PCT status may help to assess the risk for adverse renal outcomes in septic and non-septic populations.

\section{Methods}

\section{Study population}

We conducted a retrospective cohort study of adult patients consecutively admitted to a multidisciplinary ICU from June 2016 through February 2018. Critically ill patients were included in the study if they had [TIMP$2] \times$ [IGFBP7] and PCT measurements on ICU admission. We excluded patients who were in the ICU for less than $24 \mathrm{~h}$ or did not have sufficient clinical information available.

The study was approved by local Ethics Committee of the San Bortolo Hospital (Vicenza), with protocol number of 03/17, and conformed to the Declaration of Helsinki. Informed consent was obtained under Italian laws.

\section{Definitions}

Baseline serum creatinine ( $\mathrm{SCr}$ ) level was defined as the most recent SCr during 3-6 months before admission when available. In case of lacking data, the baseline $\mathrm{SCr}$ was considered admission SCr [8]. Back-estimation formula was not considered because in patients with suspected CKD, it overestimates the incidence of AKI [9].

AKI was defined and staged according to KDIGO 2012 consensus guidelines as follows: stage 1 as an increase in $\mathrm{SCr}$ level by $\leq 0.3 \mathrm{mg} / \mathrm{dL}$ ), or an increase in SCr level 1.51.9 times the baseline value or urine volume $<0.5 \mathrm{~mL} /$ $\mathrm{kg} / \mathrm{h}$ for 6-12 h; stage 2 as an increase in SCr level 2.02.9 times the baseline value or urine volume $<0.5 \mathrm{~mL} /$ $\mathrm{kg} / \mathrm{h}$ for $\geq 12 \mathrm{~h}$; stage 3 as an increase in $\mathrm{SCr}$ level 3.0 times baseline or $\geq 4.0 \mathrm{mg} / \mathrm{dL}$, or initiation of renal replacement therapy (RRT) [10].

AKD was defined as a condition in which AKI stage 1 or greater was present 7 days after an AKI initiating event [11], excluding patients who died or were discharged from the hospital within the observation period. AKD staging was defined as follows: stage 0 as a $\mathrm{SCr}$ level $<1.5$ times baseline but not back to baseline levels; stage 1 as a SCr level 1.5-1.9 times baseline; stage 2 as SCr level 2.02.9 times baseline; stage 3 as $\mathrm{SCr}$ level 3.0 times baseline or ongoing need for RRT.

Sepsis was defined according to Sepsis-III consensus definition as an acute change in total Sequential Organ Failure Assessment (SOFA) score $\geq 2$ points subsequent to an infection [12]. The baseline SOFA score was assumed to be zero in patients not known to have preexisting organ dysfunction. Infection status was defined as present in the subsequent conditions: (1) critically ill patients with confirmed infection (infective cause as reason of ICU admission); (2) critically ill patients with suspected infection in those who received empirical antibiotics after collections of body fluids for microbiological sampling within $24 \mathrm{~h} \mathrm{[13].}$

[TIMP-2] $\times$ [IGFBP7] value $>0.3(\mathrm{ng} / \mathrm{mL})^{2} / 1000$ was considered positive while a value $\leq 0.3$ negative [14] .

A PCT value $>0.5 \mu \mathrm{g} / \mathrm{L}$ was considered positive while a value $\leq 0.5$ negative.

\section{Measurements and data collection}

Blood and urine samples were collected immediately after enrollment and analysed to measure serum creatinine and urinary [TIMP-2] $\times$ [IGFBP7] concentrations, respectively. Blood samples were also collected from day 1 to day 7 of ICU stay in order to measure $\mathrm{SCr}$ concentrations.

Serum creatinine was measured using the enzymatic method with an automatic analyser (Dimension Vista, Siemens Healthcare, Tarrytown, NY). Urinary [TIMP2] $\times$ [IGFBP7] was analysed using Astute $140 \mathrm{~m}$ (Astute Medical), which divides the concentrations of the two biomarkers by 1000 to report a single numerical value in units of $(\mathrm{ng} / \mathrm{mL})^{2} / 1000$. 
Quantitative analysis of procalcitonin was performed using BRAHMS PCT sensitive KRYPTOR (ThermoFisher Scientific, Hennigsdorf, Germany). All laboratory tests were performed blinded to the clinical data.

For all patients, investigators collected from electronic health records baseline demographics, including age, sex, body mass index, comorbidities (hypertension and diabetes mellitus), reason for ICU admission, RRT requirement, duration of ICU care, duration of hospital care, duration of mechanical ventilation and vasopressors need laboratory findings and markers of clinical status (lactates levels, mean arterial pressure, diuresis). The glomerular filtration rate was estimated using the MDRD formula (eGFR) on admission. Severity of illness was calculated using the SOFA score and Simplified Acute Physiology score 2 (SAPS 2) to predict ICU mortality.

\section{Statistical analyses}

The primary endpoint was to evaluate the predictive ability of AKI development within $48 \mathrm{~h}$ for results of [TIMP$2] \times[$ IGFBP7] and PCT, alone and combined. Secondary endpoint was to assess the utility of combining the two biomarkers results for risk assessment of AKI within $48 \mathrm{~h}, \mathrm{AKD}$ at 7 days and mortality within 7 days. We also examined primary and secondary endpoints in septic and non-septic subgroups.

Continuous data were analysed using Mann-Whitney tests for non-normal data. Categorical data were analysed using Pearson Chi-square test as appropriate.

A receiver operating characteristic curve (ROC) was created for [TIMP-2] $\times$ [IGFBP7], PCT and the combination of the two biomarkers plotting the probabilities from regression model [15]. Using Youden's test, we determined an optimal cut-off and analysed its sensitivity and specificity. We also evaluated [TIMP-2] $\times$ [IGFBP7] with a validated cut-off of 0.3 and PCT with a clinical cut-off of 0.5 , analysing their sensitivity, specificity, positive and negative predictive values. Logistic regression analysis was performed to assess odds ratio (OR) of AKI occurrence within $48 \mathrm{~h}$ and results were adjusted for variables resulted significant in backward stepwise likelihood ratio model.

In order to evaluate the utility of adding [TIMP$2] \times[$ IGFBP7] and PCT results, we assess the risk for the single-biomarker positivity (when [TIMP-2] $\times$ [IGFBP7] or PCT resulted positive) and the double-biomarker positivity (when both biomarkers tested positive) relative to the negativity of both biomarkers.

All results yielding a $p$ value less than 0.05 were determined to be statistically significant.

Data were analysed using SPSS version 22.0 (IBM Corporation, Armonk, NY).

\section{Results \\ Patients characteristics}

Flowchart of the study design is shown in Fig. 1. During the study period, 507 adult ICU patients had [TIMP$2] \times$ [IGFBP7] and PCT on admission. One hundred seventy-one patients were excluded: 17 with anuria, 10 with no data available and 47 with ICU stay less than $24 \mathrm{~h}$.

Among 433 patients eligible for analysis, 181 were included in septic and 252 in non-septic cohorts. A documented baseline creatinine was present in 288 patients and admission creatinine was used as reference

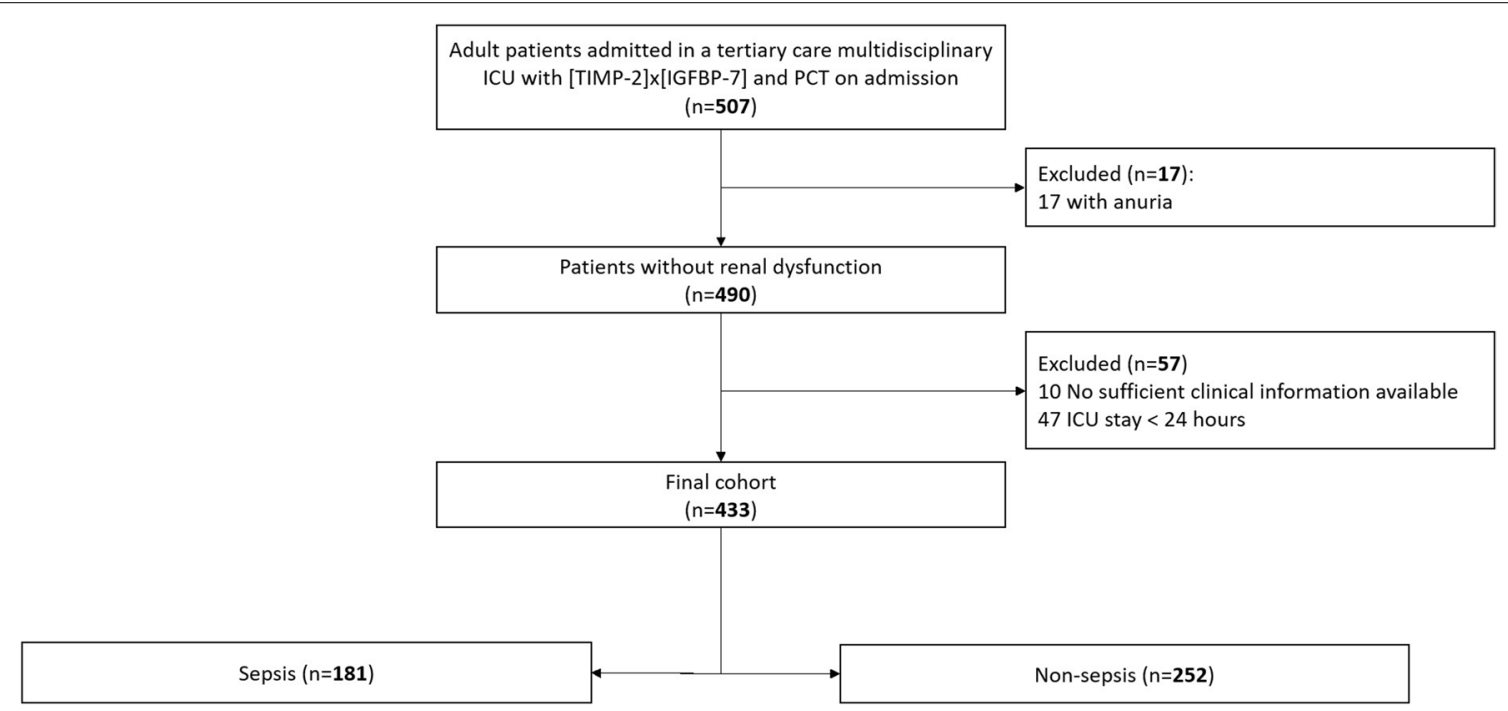

Fig. 1 Flowchart of study design. The chart shows patient inclusion and exclusion in the study analysis. ICU: intensive care unit; [TIMP-2] × [IGFBP7]: urinary tissue inhibitor of metalloproteinases-2 and insulin-like growth factor binding protein 7 product 
Table 1 Demographic data and admission characteristics

\begin{tabular}{|c|c|c|c|c|}
\hline & Analysis cohort & No AKI & AKI & $p$-value \\
\hline Patients & 433 & 265 & 168 & \\
\hline Age (years) & $70(56-78)$ & $69(54-78)$ & $72(60-80)$ & $0.042^{*}$ \\
\hline Male gender & $272(62.8)$ & $164(61.9)$ & $108(64.3)$ & $0.018^{*}$ \\
\hline $\mathrm{BMI}$ & $25(23-29)$ & $25(23-28)$ & $26(23-31)$ & $<0.001^{*}$ \\
\hline Admission reason & & & & $0.001^{*}$ \\
\hline Infection & 85 (19.6) & $35(13.2)$ & $50(29.8)$ & \\
\hline Trauma & $70(16.2)$ & $50(18.9)$ & $20(11.9)$ & \\
\hline Postoperative care & $68(15.7)$ & $41(15.5)$ & $27(16.1)$ & \\
\hline Neurologic & $61(14.1)$ & $51(19.2)$ & $10(6.0)$ & \\
\hline Cardiovascular & $55(12.7)$ & $35(13.2)$ & $20(11.9)$ & \\
\hline Respiratory & $48(11.1)$ & $29(10.9)$ & $19(11.3)$ & \\
\hline Others & $46(10.6)$ & $24(9.1)$ & $22(13.1)$ & \\
\hline \multicolumn{5}{|l|}{ Comorbidities } \\
\hline Hypertension & $226(52.2)$ & $126(47.5)$ & $100(59.5)$ & $0.015^{*}$ \\
\hline Chronic kidney disease & $53(12.2)$ & $8(3.0)$ & $45(26.8)$ & $<0.001^{*}$ \\
\hline Diabetes mellitus & $80(18.5)$ & $39(14.7)$ & $41(24.4)$ & $0.011^{*}$ \\
\hline SOFA score & $7(5-10)$ & $6(4-9)$ & $8(6-11)$ & $<0.001^{*}$ \\
\hline SAPS2 score & $50(36-63)$ & $38(27-50)$ & $42(27-53)$ & 0.375 \\
\hline Invasive ventilation time (days) & $4(2-10)$ & $3(1-10)$ & $4(2-9)$ & $0.038^{*}$ \\
\hline Vasopressor time (days) & $1(0-1)$ & $1(0-1)$ & $1(0-2)$ & $<0.001^{*}$ \\
\hline $\mathrm{eGFR}\left(\mathrm{mL} / \mathrm{min} / 1.73 \mathrm{~m}^{2}\right)$ & $81(59-99)$ & $89(70-104)$ & $68(43-89)$ & $<0.001^{*}$ \\
\hline Mean arterial pressure (mmHg) & $79(68-90)$ & $80(70-104)$ & $73(65-86)$ & $<0.001^{*}$ \\
\hline Lactates (mmol/L) & $2.0(1.4-3.5)$ & $1.7(1.2-2.6)$ & $2.6(1.7-5.1)$ & $<0.001^{*}$ \\
\hline Baseline creatinine $(\mathrm{mg} / \mathrm{dL})$ & $0.83(0.66-1.08)$ & $0.77(0.62-0.95)$ & $0.98(0.77-1.35)$ & $<0.001^{*}$ \\
\hline Urinary [TIMP-2] $\times[\mid G F B P 7](n g / m L) \wedge 2 / 1000$ & $0.5(0.1-2.1)$ & $0.22(0.07-0.99)$ & $1.39(0.47-5.72)$ & $<0.001^{*}$ \\
\hline Procalcitonin (mg/L) & $0.5(0.1-4.6)$ & $0.2(0.1-0.9)$ & $3.2(0.6-17.6)$ & $<0.001^{*}$ \\
\hline
\end{tabular}

Demographic data and admission characteristics. Data are reported as numbers (percentages) as categorical variables and median (interquartile range) for continuous variables. * identified a $p$-value $<0.05$

$B M I$ body mass index, SOFA Sequential Organ Failure Assessment, SAPS2 Simplified Acute Physiology score 2, eGFR estimated glomerular filtration rate, [TIMP$2] \times[I G F B P 7]$ tissue inhibitor metalloproteinase 2 and insulin-growth factor binding protein 7 product

creatinine in 145 patients. Basal characteristics are described in Table 1.

[TIMP-2] $x$ [IGFBP7] and PCT levels at the time of admission and AKI development

Of the 433 patients, 168 (38.8\%) developed AKI within $48 \mathrm{~h}$, of which $55(12.7 \%)$ were categorized as stage 1, $44(10.2 \%)$ as stage 2 and $69(15.9 \%)$ as stage 3 (Table 2 ). Patients with AKI at ICU admission were $6.0 \%(n=26)$, $28.4 \%(n=123)$ had AKI between admission and $24 \mathrm{~h}$, and $4.4 \%(n=19)$ had AKI between 24 and $48 \mathrm{~h}$.

Patients with AKI within $48 \mathrm{~h}$ were older (72 vs 69 years, $p=0.042$ ), with more acute critical illness (SOFA score 8 vs $6, p<0.001$ ), with a prevalence of infective cause for ICU admission. $59.5 \%$ of them had a history of hypertension, $24.4 \%$ diabetes mellitus and $26.8 \%$ chronic kidney disease. These patients also had lower eGFR (89 vs $68 \mathrm{~mL} / \mathrm{min} / 1.73 \mathrm{~m}^{2}, p<0.001$ ) and mean arterial pressure (80 vs $73 \mathrm{mmHg}, p<0.001)$ on admission, higher lactate levels (1.7 vs $2.6 \mathrm{mmol} / \mathrm{L}$, $p<0.001$ ) and needed mechanical ventilation (3 vs 4 days, $p=0.038)$ and vasopressors for longer period (1 [0-1] vs 1 [0-2] days, $p<0.001)$. [TIMP-2] $\times$ [IGFBP7] values were higher in AKI patients compared to those who did not develop AKI (0.63 [0.13-2.19] vs 0.23 [0.06-0.59], $p<0.001)$, as well as PCT $(1.20[0.20-6.70]$ vs $0.20[0.10-$ $0.90], p<0.001)$.

In order to evaluate the biomarkers predictive ability for AKI occurrence, we performed a ROC curve analysis. [TIMP-2] $\times$ [IGFBP7] showed a fair predictive ability for AKI development (AUC 0.75, Sens 83\%, Spec 59\%), as well as PCT (AUC 0.79, Sens 79\%, Spec 67\%). The combination of the two biomarkers slightly increased the predictive ability for AKI occurrence (AUC 0.81, 95\% CI $0.77-0.86, p<0.001$, Sens $78 \%$, Spec $73 \%$ ). In the overall population, the optimal cut-offs of [TIMP-2] $\times$ [IGFBP7] were higher than the validated cut-off of 0.3 and showed higher specificity, while optimal cut-off of PCT 
Table 2 Patients' outcomes in the overall population and in septic and non-septic subgroups

\begin{tabular}{|c|c|c|c|c|}
\hline & Analysis cohort & Sepsis & Non-sepsis & $p$-value \\
\hline Patients & 433 & 181 & 252 & \\
\hline $\begin{array}{l}\text { AKI at ICU admis- } \\
\text { sion }\end{array}$ & $26(6.0)$ & $14(7.7)$ & $12(4.8)$ & $0.001^{*}$ \\
\hline Stage 1 & $14(3.2)$ & $8(4.4)$ & $6(2.4)$ & \\
\hline Stage 2 & $5(1.1)$ & $2(1.1)$ & $3(1.2)$ & \\
\hline Stage 3 & $7(1.6)$ & $4(2.2)$ & $3(1.2)$ & \\
\hline AKI within $24 \mathrm{~h}$ & $149(34.4)$ & $82(45.3)$ & $67(26.6)$ & $<0.001^{*}$ \\
\hline Stage 1 & 46 (10.6) & 25 (13.8) & $21(8.3)$ & \\
\hline Stage 2 & $38(8.8)$ & 19 (10.5) & $19(7.5)$ & \\
\hline Stage 3 & 65 (15.0) & $38(21.0)$ & 27 (10.7) & \\
\hline AKI within $48 \mathrm{~h}$ & $168(38.8)$ & $93(51.4)$ & $75(29.8)$ & $<0.001^{*}$ \\
\hline Stage 1 & $55(12.7)$ & $31(17.1)$ & $24(9.5)$ & \\
\hline Stage 2 & $44(10.2)$ & $22(12.2)$ & $22(8.7)$ & \\
\hline Stage 3 & $69(15.9)$ & $40(22.1)$ & $29(11.5)$ & \\
\hline RRT need & $33(7.6)$ & $18(9.9)$ & $15(6.0)$ & \\
\hline AKD at 7 days & $47(10.8)$ & $26(14.4)$ & $21(8.3)$ & $<0.01^{*}$ \\
\hline Stage 0 & $15(3.5)$ & $10(5.5)$ & $5(2.0)$ & \\
\hline Stage 1 & $9(2.1)$ & $5(2.8)$ & $4(1.6)$ & \\
\hline Stage 2 & $7(1.6)$ & $4(2.2)$ & $3(1.2)$ & \\
\hline Stage 3 & $16(3.7)$ & $7(3.9)$ & 9 (3.6) & \\
\hline RRT need & $14(3.2)$ & $7(3.9)$ & $7(2.8)$ & \\
\hline 7 days mortality & 65 (15.0) & $37(20.4)$ & $28(11.1)$ & $<0.01^{*}$ \\
\hline ICU mortality & $100(23.1)$ & $50(27.6)$ & $50(19.8)$ & 0.57 \\
\hline Days in ICU & $4(2-11)$ & $4(2-10)$ & $4(2-11)$ & 0.77 \\
\hline Hospital mortality & $128(29.6)$ & $64(35.4)$ & 64 (25.4) & 0.37 \\
\hline Days in hospital & $15(7-31)$ & $13(6-30)$ & $15(7-31)$ & 0.27 \\
\hline
\end{tabular}

Data are reported as numbers (percentages) as categorical variables and median (interquartile range) for continuous variables. * identified a $p$-value $<0.05$

$A K I$ acute kidney injury, $A K D$ acute kidney disease, $R R T$ renal replacement therapy, ICU intensive care unit

approximated the clinical one. The results of ROC curve analysis (AUCs, optimal cut-offs, Sens and Spec) for the entire cohort and for subgroups are detailed in Additional file 1: Table S1 and Additional file 2: Table S2.

AKI risk assessment by [TIMP-2] x [IGFBP7] and PCT results and their combination

Logistic regression analysis was performed to assess odds ratio (OR) of AKI occurrence within $48 \mathrm{~h}$ using the validated cut-off of 0.3 for [TIMP-2] $\times$ [IGFBP7] and the widely used cut-off of 0.5 for PCT. After multivariate analysis which incorporated admission eGFR and SOFA score, the positivity of the biomarkers was associated with an increased risk for AKI occurrence (Table 3). Of note, $\mathrm{PCT}>0.5$ was not an independent predictor of AKI in septic population. To evaluate the utility of adding [TIMP-2] $\times$ [IGFBP7] and PCT results for risk assessment, we evaluated the predictive value of having a single-marker positivity compared to a double-marker positivity. As shown in Table 3, the presence of at least one of the two biomarkers was significantly associated with AKI development (OR 4.1, 95\% CI 1.9-8.8, $p<0.001$ ) and when both biomarkers were positive the probability of AKI occurrence strongly increased (OR 26.4, 95\% CI 12.3-56.62, $p<0.001)$. If the results were confirmed in non-septic population, within septic patients only the positivity of both biomarkers significantly increased the risk for AKI occurrence with a 19-fold risk increase.

\section{Association of combined positivity of [TIMP-2] x [IGFBP7] and PCT with AKD at 7 days}

To determine a potential association with persistence of AKI, a subset analyses was performed excluding patients who died or were discharged from the hospital within the observation period $(n=136) .47$ of 297 patients had AKD at 7 days (15.8\%), of whom 14 were classified in stage 0 ( $n=26$ in septic group), 9 in stage 1 ( $n=5$ in septic group), 7 in stage 2 ( $n=4$ in septic group), 16 in stage 3 ( $n=7$ in septic group) and 14 required RRT ( $n=7$ in septic group). As shown in Table 3, a double-biomarker positivity had a 10.3 risk increase for AKD at 7 days $(p=0.001)$. These results were confirmed in non-septic population, where the risk for AKD was 15.9 times higher when both biomarkers tested positive.

\section{Association of combined positivity of [TIMP-2] x [IGFBP7] and PCT with mortality within 7 days}

Of the 433 patients analysed, 65 died within 7 days ( $n=37$ in septic group). Using Cox regression analysis, only the double-marker positivity showed an increased risk for mortality within 7 days (Table 3 ) and these results were confirmed only in septic patients (HR 4.1, 95\% CI $1.4-11.8, p=0.001$ ).

\section{Discussion}

AKI is a commonly encountered complication of critical illness, associated with significant mortality, long-term adverse outcomes and high costs [16]. AKI and its progression could be prevented or mitigated by effective care strategies if sufficient warning is provided [10]. Sepsis is one of the most common causes of AKI in critical care patients and the two syndromes can interweave in the condition of sepsis-associated AKI [17].

Biomarkers are potential tools that offer early warning where intervention can still alter outcomes. Some biomarkers revealed activity in biological pathways that precede AKI and other biomarkers are triggered by the presence of subclinical AKI. Therefore, combining the results of biomarkers involved in different steps of disease process may be helpful in the prediction and assessment of the risk. 
Table 3 Risk assessment for primary and secondary outcomes in the entire population and in septic and non-septic subgroups

\begin{tabular}{|c|c|c|c|c|c|c|c|c|c|}
\hline \multirow[t]{2}{*}{ Variables } & \multicolumn{3}{|c|}{ Analysis cohort } & \multicolumn{3}{|c|}{ Sepsis } & \multicolumn{3}{|c|}{ Non-sepsis } \\
\hline & OR & $95 \% \mathrm{Cl}$ & $p$-value & OR & $95 \% \mathrm{Cl}$ & $p$-value & OR & $95 \% \mathrm{Cl}$ & $p$-value \\
\hline \multicolumn{10}{|l|}{ Primary outcome } \\
\hline \multicolumn{10}{|l|}{ AKI within $48 \mathrm{~h}$} \\
\hline$[\mathrm{TIMP}-2] \times[\mid \mathrm{GFBP} 7]>0.3$ & $3.93^{\mathrm{a}}$ & $2.14-7.20$ & $<0.001$ & $5.92^{\mathrm{a}}$ & $2.53-13.82$ & $<0.001$ & $3.27^{\mathrm{a}}$ & $1.53-6.97$ & $<0.001$ \\
\hline $\mathrm{PCT}>0.5$ & $3.67^{\mathrm{a}}$ & $2.17-6.19$ & $<0.001$ & $2.74^{\mathrm{a}}$ & $0.87-8.57$ & 0.083 & $4.85^{\mathrm{a}}$ & $2.37-9.94$ & $<0.001$ \\
\hline Single-marker positivity & 4.08 & $1.90-8.76$ & $<0.001$ & 2.27 & $0.45-11.23$ & 0.316 & 4.93 & $2.05-11.82$ & $<0.001$ \\
\hline Double-marker positivity & 26.41 & $12.32-56.62$ & $<0.001$ & 19.5 & $4.20-90.44$ & $<0.001$ & 25.11 & $9.58-65.79$ & $<0.001$ \\
\hline \multicolumn{10}{|l|}{ Secondary outcomes } \\
\hline \multicolumn{10}{|l|}{ AKD at 7 days } \\
\hline Single-marker positivity & 4.73 & $1.04-21.60$ & 0.045 & 2.28 & $0.26-20.02$ & 0.15 & 3.24 & $0.65-16.20$ & 0.151 \\
\hline Double-marker positivity & 15.92 & $3.67-68.97$ & 0.001 & 4.57 & $0.48-36.25$ & 0.15 & 15.36 & $3.21-73.57$ & 0.001 \\
\hline \multicolumn{10}{|l|}{ Mortality within 7 days } \\
\hline Single-marker positivity & 1.16 & $0.51-2.65$ & 0.724 & 0.86 & $0.77-2.75$ & 0.494 & 1.59 & $0.63-4.05$ & 0.329 \\
\hline Double-marker positivity & 2.75 & $1.34-5.65$ & 0.006 & 4.1 & $1.41-11.78$ & 0.001 & 2.01 & $0.75-5.40$ & 0.166 \\
\hline
\end{tabular}

A single-marker positivity was defined by the presence of [TIMP-2] $\times$ [IGFBP7] above the cut-off of 0.3 or PCT above the cut-off of 0.5 ; the double-marker positivity was defined by the presence of [TIMP-2] $\times$ [IGFBP7] measurements above 0.3 and the concomitant presence of PCT levels above 0.5

[TIMP-2] $\times$ [IGFBP7] tissue inhibitor metalloproteinase-2 and insulin-growth factor binding protein 7 product, $P C T$ procalcitonin

a Odds ratios (OR) were adjusted for Sequential Organ Failure Assessment (SOFA) and estimated glomerular filtration rate (eGFR) at the time of admission

The [TIMP-2] $\times$ [IGFBP7] product is known to be a biomarker of kidney stress in multiple settings [18], while PCT is recognized as a suitable marker for sepsis (or infection) diagnosis and has recently been associated with AKI (contrast-induced AKI [19] and pancreatitisassociated AKI [20] and critically ill patients [7]).

Some characteristics may link PCT and [TIMP$2] \times$ [IGFBP7] in AKI and in septic AKI. First, both biomarkers can be upregulated by ROS in the context of hypoperfusion and hypoxia. ROS production have been shown to induce PCT expression [21] and to be a potential stress insult to induce TIMP-2 and IGFBP7 production [22].

Second, both biomarkers might be expression of an inflammatory status, more or less kidney-specific. In vitro results demonstrated that peripheral blood mononuclear cells (PBMCs), as well as endothelial cells, were potentially capable of contributing to in vivo extrathyroidal PCT production [23]. Activated PBMCs and proinflammatory cytokines, such as tumour necrosis factor alpha and interleukin 1 are considered to play a role in PCT production and inflammation [24]. In a recent study by Gocze et al., a tubular stress detected by [TIMP$2] \times$ [IGFBP7] positivity was associated with a systemic $\gamma \delta \mathrm{T}$ cell immune cell response [25].

The main findings of this study were the subsequent: (1) the combination of the biomarkers demonstrated a good predictive ability for patients at risk for AKI occurrence; (2) combined results according to widely used cut-offs of 0.3 for [TIMP-2] $\times$ [IGFBP7] and 0.5 for PCT allowed risk stratification of the population for AKI development within $48 \mathrm{~h}$; (3) A double-marker positivity, defined by the concomitant positivity of both biomarkers was also significantly associated with mortality within 7 days in septic subgroup and with AKD at 7 days in nonseptic patients.

Several clinical studies have evaluated the utility of [TIMP-2] $\times$ [IGFBP7] product in the early diagnosis and risk stratification of AKI $[5,26]$. Honore et al. found that [TIMP-2] $\times$ [IGFBP7] accurately predicted AKI in septic patients [27]. Our study found a predictive ability of [TIMP-2] $\times$ [IGFBP7] for AKI in critically ill and septic patients lower than previously described. Furthermore, the cut-off proposed by Hoste et al. [14] showed a good sensitivity, but a low negative predictive value. These findings probably reflected the heterogeneity of our ICU population as well as the study design. It should be taken also into account that the primary endpoint was development of any stage of AKI. Surprisingly, PCT showed a better predictive ability compared to cell cycle arrest biomarkers, both in septic and non-septic groups.

Literature proved PCT to be a strong marker of infection and sepsis and recently PCT have been significantly associated with subsequent AKI development in critically ill patients [7]. Despite sepsis, other noninfective conditions, such as surgery, trauma, burn, pancreatitis, renal dysfunction can also increase PCT levels [28]. In some of our patients, PCT was elevated regardless of their 
non-septic condition and the most common admission reason was trauma. Other admission causes were postoperative care, neurological, cardiovascular and respiratory causes. Even though the association between PCT and AKI exists, the usefulness of adding PCT levels to the existing models is still not clear for predicting the occurrence of AKI in critically ill patients in the intensive care unit (ICU) and the interpretation of PCT elevation on admission as signal of AKI requires caution.

Therefore, we investigated the combination of [TIMP$2] \times$ [IGFBP7] and PCT for AKI prediction with the hypothesis that the addition of an AKI biomarker with a sepsis biomarker may lead to early identification of patients with sepsis-induced AKI in order to direct preventive or proactive strategies and preserve renal function. Our results demonstrate that combining biomarkers enabled enhanced risk prediction by way of a composite risk assessment. Furthermore, using the validated cut-off of 0.3 for [TIMP-2] $\times$ [IGFBP7] and the widely used cutoff of 0.5 for PCT, we can stratify patients according to the presence of single or double-biomarker positivity. The combined positivity of the two biomarkers had approximately 26-fold increase of AKI risk, which represents a very large increase in OR from 4.0 when only one of the two biomarkers was positive. Whether this increase might be considered clinically relevant will depend on the baseline risk of the patients. Interestingly, these results were confirmed in septic and non-septic patients. The findings that [TIMP-2] $\times$ [IGFBP7] and PCT combined increased the risk for AKI also in non-septic patients led to considering two possible hypotheses. Firstly, PCT a surrogate marker of sepsis; in this line, the combination of the two biomarkers may help in risk prediction and stratification for AKI and sepsis and may highlight [TIMP-2] $\times$ [IGFBP7] to be not only an AKI biomarker, but also a marker of sepsis. Previous trials studied PCT as a marker of sepsis after surgery and trauma, however evidence is lacking $[29,30]$. A second hypothesis may be the association between inflammation and subsequent AKI development. In consideration of that our ICU policy does not provide a routine use of PCT and the measurements were requested according to clinicians' judgement, therefore our population have to be considered at risk for infection and inflammation. Nonetheless, we cannot conclude for any of the two hypothesis, because other markers of inflammation or infection should be evaluated.

A recent study also investigated urinary cell cycle arrest biomarkers in conjunction with classical markers of AKI to improve risk stratification for short-term severe outcomes [31]. PCT was found to be correlated with mortality only when serial measurements were performed [32]. We found that the combined positivity of [TIMP$2] \times[$ IGFBP7 $]$ and PCT was associated with an increased risk for mortality within 7 days in septic patients and for $\mathrm{AKD}$ at 7 days in non-septic patients. We also found that the combined positivity reflected a more critical pattern both in septic and non-septic groups. For instance, the positivity of both biomarkers was associated with increased risk of mortality within 7 days, possibly reflecting the most critically ill patients, especially those with confirmed or suspected sepsis. From the other side the double-marker positivity combination at the time of admission was associated with an increased risk for AKD at 7 days in non-septic patients, suggesting that a septic/ inflammatory state increased the risk for persistence of the renal insult. This is the first study to explore the relationship between combination of urinary cell cycle arrest biomarkers and PCT and short-term outcomes in septic and non-septic critically ill patients.

Our study has several limitations. First, this is a retrospective analysis with a small sample size in a single centre. As a secondary analysis of data from a prospective observational study, available information is limited. We have no information on the focus of infection and pathogens detected. Second, the fact that PCT levels may be affected by either acute or chronic renal dysfunction is under debate [24,33] and the diagnostic accuracy of PCT level may be lower in patients with AKI or CKD. On the other hand, selection bias should be taken into account because of the exclusion criteria used. Of note, for research purpose [TIMP-2] $\times$ [IGFBP7] was measured in all patients admitted to our ICU during the study period. The results were therefore adjusted for factors that were linked to the endpoints. Third, we performed only baseline measurements of biomarkers under investigation and cannot clarify the variability in biomarkers during the study, whilst ICU patient's status may change dramatically within hours. Fourth, AKI within 24 and $48 \mathrm{~h}$ may be viewed as a debatable timeframe; indeed, some patients may develop AKI afterwards. In addition, AKD was handled as a non-time-dependent variable, whereas this variable is strongly affected by time dependency, even though we tried to limit this bias by excluding from analysis those patients who died or were discharged from the hospital.

Despite such limitations, the study might be relevant for clinical implication and further research on sepsis and AKI field.

\section{Conclusions}

[TIMP-2] $\times$ [IGFBP7] and PCT can be used to identify critically ill patients at risk for AKI occurrence. The combination of [TIMP-2] $\times$ [IGFBP7] and PCT with cut-offs of 0.3 and 0.5 , respectively, may help in stratifying the risk for AKI, regardless of sepsis. The present study also showed that the combined biomarkers elevation over the 
cut-off values was a predictor for mortality within 7 days in patients with suspected or confirmed sepsis and for AKD at 7 days within patients without sepsis.

Although combining the results of [TIMP$2] \times[$ IGFBP7] and PCT could be in the future a useful tool for assessing the risk for septic AKI and short-term adverse outcomes, data should be confirmed in a large prospective study.

\section{Supplementary information}

Supplementary information accompanies this paper at https://doi. org/10.1186/s13613-020-00665-9.

Additional file 1. ROC curve analysis for [TIMP-2] × [IGFBP7], procalcitonine and the combination of the two biomarkers.

Additional file 2. ROC curve analysis for [TIMP-2] × [IGFBP7] and procalcitonine with predefined cut-offs of 0.3 and 0.5 respectively, and the combination of the two results.

\section{Abbreviations}

AKI: Acute kidney injury; AKD: Acute kidney disease; CKD: Chronic kidney disease; HR: Hazard ratio; ICU: Intensive care unit; IQR: Interquartile range; OR: Odds ratio; PCT: Procalcitonin; SAPS2: Simplified Acute Physiology score 2; SCr: Serum creatinine; SOFA: Sequential Organ Failure Assessment; [TIMP2] $\times$ [IGFBP7]: Urinary tissue inhibitor metalloproteinase-2 and insulin-like growth factor binding protein 7 product.

\section{Acknowledgements}

The authors are grateful to all the fellows of the International Renal Research Institute of Vicenza (IRRIV), the staff, physicians and nurses at the ICU and Nephrology Department of San Bortolo Hospital in Vicenza for the hard work.

\section{Authors' contributions}

IG, SDR, FM and CR contributed to study design. IG, SB, CTD, GM, AL, MdC, VC and CC contributed to data collection. SDR, MM, EB contributed to patient enrolment. MRC and DG contributed to laboratory work. IG and FM contributed to statistical analysis. IG, SDR and CR contributed to manuscript writing and editing. All authors reviewed the final manuscript. All authors read and approved the final manuscript.

\section{Funding}

Not applicable.

\section{Availability of data and materials}

All data analysed during the current study are available from the corresponding author on reasonable request.

\section{Ethics approval and consent to participate}

The original study was approved by the institutional ethics committee of San Bortolo Hospital in Vicenza, Italy (Comitato Etico provinciale ULSS 8 Berica) (Protocol number: 03/17). The clinical investigation was conducted according to the principles expressed in the Declaration of Helsinki.

\section{Consent for publication}

Not applicable.

\section{Competing interests}

The authors declare no competing interests.

\section{Author details}

${ }^{1}$ International Renal Research Institute of Vicenza (IRRIV), San Bortolo Hospital, Vicenza, Italy. ${ }^{2}$ Department of Medicine-DIMED, Section of Anesthesiology and Intensive Care Medicine, University of Padova, Padua, Italy. ${ }^{3}$ Department of Anesthesiology and Intensive Care Medicine, San Bortolo Hospital, Vicenza, Italy. ${ }^{4}$ Department of Nephrology, Dialysis and Transplantation, San Bortolo
Hospital, Vicenza, Italy. ${ }^{5}$ Department of Anesthesiology and Intensive Care, Azienda Ospedalieriero-Universitaria Maggiore DELLA Carità, Novara, Italy.

${ }^{6}$ Department of Laboratory Medicine, San Bortolo Hospital, Vicenza, Italy.

Received: 23 September 2019 Accepted: 13 April 2020

Published online: 21 April 2020

\section{References}

1. Hoste EAJ, Kellum JA, Selby NM, Zarbock A, Palevsky PM, Bagshaw SM, et al. Global epidemiology and outcomes of acute kidney injury. Nat Rev Nephrol. 2018;14(10):607-25 (Epub 2018/08/24).

2. Peters E, Antonelli M, Wittebole X, Nanchal R, Francois B, Sakr Y, et al. A worldwide multicentre evaluation of the influence of deterioration or improvement of acute kidney injury on clinical outcome in critically ill patients with and without sepsis at ICU admission: results from The Intensive Care Over Nations audit. Crit Care. 2018;22(1):188 (Epub 2018/08/05)

3. Lewington AJ, Cerda J, Mehta RL. Raising awareness of acute kidney injury: a global perspective of a silent killer. Kidney Int. 2013;84(3):457-67 (Epub 2013/05/03).

4. Ostermann M, Cerda J. The burden of acute kidney injury and related financial issues. Contrib Nephrol. 2018;193:100-12 (Epub 2018/02/03).

5. Kashani K, Al-Khafaji A, Ardiles T, Artigas A, Bagshaw SM, Bell M, et al. Discovery and validation of cell cycle arrest biomarkers in human acute kidney injury. Crit Care. 2013;17(1):25 (Epub 2013/02/08).

6. Jeeha R, Skinner DL, De Vasconcellos K, Magula NP. Serum procalcitonin levels predict acute kidney injury in critically ill patients. Nephrology. 2018;23(12):1090-5 (Epub 2017/10/03).

7. Chun K, Chung W, Kim AJ, Kim H, Ro H, Chang JH, et al. Association between acute kidney injury and serum procalcitonin levels and their diagnostic usefulness in critically ill patients. Sci Rep. 2019;9(1):4777 (Epub 2019/03/20).

8. Thomas ME, Blaine C, Dawnay A, Devonald MA, Ftouh S, Laing C, et al. The definition of acute kidney injury and its use in practice. Kidney Int. 2015;87(1):62-73 (Epub 2014/10/16).

9. De Rosa S, Samoni S, Ronco C. Creatinine-based definitions: from baseline creatinine to serum creatinine adjustment in intensive care. Crit Care. 2016;20:69 (Epub 2016/03/18)

10. Khwaja A. KDIGO clinical practice guidelines for acute kidney injury. Nephron Clinical practice. 2012;120(4):c179-84 (Epub 2012/08/15).

11. Chawla LS, Bellomo R, Bihorac A, Goldstein SL, Siew ED, Bagshaw SM, et al. Acute kidney disease and renal recovery: consensus report of the Acute Disease Quality Initiative (ADQI) 16 Workgroup. Nat Rev Nephrol. 2017;13(4):241-57 (Epub 2017/02/28)

12. Singer $M$, Deutschman CS, Seymour CW, Shankar-Hari M, Annane D, Bauer $M$, et al. The third international consensus definitions for sepsis and septic shock (sepsis-3). JAMA. 2016;315(8):801-10 (Epub 2016/02/24).

13. Seymour CW, Liu VX, Iwashyna TJ, Brunkhorst FM, Rea TD, Scherag A, et al. Assessment of clinical criteria for sepsis: for the third international consensus definitions for sepsis and septic shock (sepsis-3). JAMA. 2016;315(8):762-74 (Epub 2016/02/24)

14. Hoste EA, McCullough PA, Kashani K, Chawla LS, Joannidis M, Shaw AD, et al. Derivation and validation of cutoffs for clinical use of cell cycle arrest biomarkers. Nephrol Dial Transpl. 2014;29(11):2054-61 Epub 2014/09/23.

15. Pepe MS, Thompson ML. Combining diagnostic test results to increase accuracy. Biostatistics. 2000;1 (2):123-40 (Epub 2003/08/23)

16. Hoste EA, Bagshaw SM, Bellomo R, Cely CM, Colman R, Cruz DN, et al. Epidemiology of acute kidney injury in critically ill patients: the multinational AKI-EPI study. Intensiv Care Med. 2015;41(8):1411-23 (Epub 2015/07/15)

17. Godin M, Murray P, Mehta RL. Clinical approach to the patient with AKI and sepsis. Semin Nephrol. 2015;35(1):12-22 (Epub 2015/03/22).

18. Jia HM, Huang LF, Zheng Y, Li WX. Diagnostic value of urinary tissue inhibitor of metalloproteinase-2 and insulin-like growth factor binding protein 7 for acute kidney injury: a meta-analysis. Crit Care. 2017;21(1):77 (Epub 2017/03/28).

19. Kurtul A, Murat SN, Yarlioglues M, Duran M, Ocek AH, Celik IE, et al. Procalcitonin as an early predictor of contrast-induced acute kidney 
injury in patients with acute coronary syndromes who underwent percutaneous coronary intervention. Angiology. 2015;66(10):957-63 (Epub 2015/02/18)

20. Clementi A, Virzi GM, Mucino-Bermejo MJ, Nalesso F, Giavarina D, Carta M, et al. Presepsin and procalcitonin levels as markers of adverse postoperative complications and mortality in cardiac surgery patients. Blood Purif. 2019:47(1-3):140-8 (Epub 2018/10/20)

21. Raddant AC, Russo AF. Reactive oxygen species induce procalcitonin expression in trigeminal ganglia glia. Headache. 2014;54(3):472-84 (Epub 2014/02/12)

22. Kellum JA, Chawla LS. Cell-cycle arrest and acute kidney injury: the light and the dark sides. Nephrol Dial Transpl. 2016;31(1):16-22 (Epub 2015/06/06)

23. Zannoni A, Giunti M, Bernardini C, Gentilini F, Zaniboni A, Bacci ML, et al. Procalcitonin gene expression after LPS stimulation in the porcine animal model. Res Vet Sci. 2012;93(2):921-7 (Epub 2011/10/18)

24. Rodriguez A, Reyes LF, Monclou J, Suberviola B, Bodi M, Sirgo G, et al. Relationship between acute kidney injury and serum procalcitonin (PCT) concentration in critically ill patients with influenza infection. Medicina Intensiva. 2018:42(7):399-408 (Epub 2018/02/13)

25. Gocze I, Ehehalt K, Zeman F, Riquelme P, Pfister K, Graf BM, et al. Postoperative cellular stress in the kidney is associated with an early systemic gammadelta T-cell immune cell response. Crit Care. 2018;22(1):168 (Epub 2018/07/06)

26. Bihorac A, Chawla LS, Shaw AD, Al-Khafaji A, Davison DL, Demuth GE, et al. Validation of cell-cycle arrest biomarkers for acute kidney injury using clinical adjudication. Am J Respir Crit Care Med. 2014;189(8):932-9 (Epub 2014/02/25).

27. Honore PM, Nguyen HB, Gong M, Chawla LS, Bagshaw SM, Artigas A, et al. Urinary tissue inhibitor of metalloproteinase-2 and insulin-like growth factor-binding protein 7 for risk stratification of acute kidney injury in patients with sepsis. Crit Care Med. 2016;44(10):1851-60 (Epub 2016/06/30)

28. Nijsten MW, Olinga P, The TH, de Vries EG, Koops HS, Groothuis GM, et al. Procalcitonin behaves as a fast responding acute phase protein in vivo and in vitro. Crit Care Med. 2000;28(2):458-61 (Epub 2000/03/09).

29. Uzzan B, Cohen R, Nicolas P, Cucherat M, Perret GY. Procalcitonin as a diagnostic test for sepsis in critically ill adults and after surgery or trauma: a systematic review and meta-analysis. Crit Care Med. 2006;34(7):19962003 (Epub 2006/05/23)

30. Parli SE, Trivedi G, Woodworth A, Chang PK. Procalcitonin: usefulness in acute care surgery and trauma. Surg Infect. 2018;19(2):131-6 (Epub 2018/01/23).

31. Joannidis M, Forni LG, Haase M, Koyner J, Shi J, Kashani K, et al. Use of cell cycle arrest biomarkers in conjunction with classical markers of acute kidney injury. Crit Care Med. 2019;47(10):e820-6 (Epub 2019/07/26).

32. Jensen JU, Heslet L, Jensen TH, Espersen K, Steffensen P, Tvede M. Procalcitonin increase in early identification of critically ill patients at high risk of mortality. Crit Care Med. 2006;34(10):2596-602 (Epub 2006/08/18).

33. Nakamura Y, Murai A, Mizunuma M, Ohta D, Kawano Y, Matsumoto N, et al. Potential use of procalcitonin as biomarker for bacterial sepsis in patients with or without acute kidney injury. J Infect Chemother. 2015;21(4):257-63 (Epub 2015/02/14).

\section{Publisher's Note}

Springer Nature remains neutral with regard to jurisdictional claims in published maps and institutional affiliations.

\section{Submit your manuscript to a SpringerOpen ${ }^{\circ}$ journal and benefit from:}

- Convenient online submission

- Rigorous peer review

- Open access: articles freely available online

- High visibility within the field

- Retaining the copyright to your article

Submit your next manuscript at $\boldsymbol{\nabla}$ springeropen.com 